\title{
A influência do exercício físico no perfil lipídico e na aptidão física em mulheres idosas
}

\author{
The influence of exercise in the lipidic profile and in the physical fitness in \\ elderly women

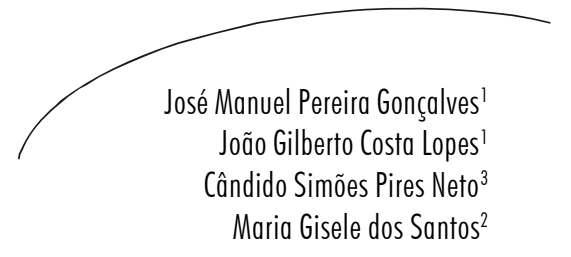

\section{Resumo}

Objetivo: Avaliar a influência do nível de atividade física no perfil lipídico, na glicose sanguínea, na composição corporal, na aptidão cardiovascular (VO2max), na aptidão muscular e na agilidade/equilíbrio em mulheres idosas. Métodos: Para verificar interferência do nível de atividade física nos níveis lipídicos, glicêmicos, de composição corporal e de aptidão física, foi utilizado o teste $t$ de Student independente, precedido do teste de normalidade de Shapiro-Wilks. Foram avaliadas 23 mulheres com mais de 60 anos, divididas em um grupo ativo (GA) e grupo inativo (GI) segundo classificação do IPAQ. Resultados: Mulheres idosas do grupo ativo apresentaram valores significativamente superiores no teste de agilidade/equilíbrio e idade média superior ao do grupo inativo. Além disso, o grupo ativo apresentou valores superiores na massa de gordura, massa corporal magra e força de membros inferiores, mas não estatisticamente significativos. De forma inversa, o colesterol total, triglicérides e a glicose sanguínea mostraram-se inferiores no grupo ativo, mas também não estatisticamente significativos. As demais variáveis antropométricas, perfil qualitativo alimentar, e demais testes de aptidão física não mostraram diferenças

Palavras-chave: Perfil de Saúde. Metabolismo dos Lipídeos. Glicemia. Composição Corporal. Aptidão Física. Fisiologia Cardiovascular. Equilíbrio Musculosquelético. Mulheres. Idosas.

1 Mayoredad Centro de Promoção da Saúde e Qualidade de Vida. Curitiba, PR, Brasil

2 Universidade Federal do Paraná, Setor de Ciências Biológicas, Departamento de Educação Física. Curitiba, PR, Brasil

3 Unidade de Ensino Superior Vale do Iguaçu, Curso de Educação Física. União da Vitória, PR, Brasil

Correspondência / Correspondence José Manuel Pereira Gonçalves Av. Prefeito Omar Sabbag, 526/03 - Jardim Botânico 80210- 000 - Curitiba, PR, Brasil

E-mail: jmpg@ibest.com.br 
entre os grupos. Conclusão: Conclui-se que a adoção e manutenção de um estilo de vida ativo, mesmo em idades avançadas, por meio da atividade física, têm efeitos benéficos na manutenção da independência de pessoas idosas.

\section{Abstract}

Objective: To value the influence of the level of physical activity in the lipid profiles, the levels of glucose in the blood stream, the body composition, the muscular and cardiovascular fitness aptitudes ( $\left.\mathrm{VO}^{2} \mathrm{max}\right)$, and the agility/ equilibrium in elderly women Methods: In order to verify the interference of the level of physical exercises on the levels of lipid and glucose, on body composition and on physical aptitude, the independent Student test was used, after the normality Shapiro-Wilks test. Twenty-three women over 60 years old were evaluated in two groups, an active group and an inactive one, according to a classification by IPAQ. Results: Active women presented significantly higher values in the agility/equilibrium tests and middle age superior to that of the inactive group. Besides, the active group presented superior fatness mass, slim body mass, strength in inferior members, which were not, however, statistically significant. In the reverse form, the total cholesterol, triglycerids and they showed the blood glucose inferior in the active group, but also not statistically significant. You were varying the rest antropométricas, qualitative food profile, and too many tests of physical aptitude did not show differences between the groups. Conclusions: The conclusion is that adopting and keeping up an active lifestyle by means of physical activities, even in advanced age, has benefic effects in prolonging the independence of the elderly.
Key words: Health Profile. Lipid Metabolism. Blood Glucose. Body Composition. Physical Fitness. Cardiovascular Physiology. Equilíbrio Musculosquelético. Women. Elderly.

\section{INTRODUÇÃO}

Em países desenvolvidos, o aumento na expectativa de vida vem sendo relacionado a importantes descobertas da medicina, além da urbanização adequada, melhoria nutricional, níveis de higiene pessoal e condições sanitárias no trabalho e nas residências. Este fato também é observado em países de Terceiro Mundo, embora a diferença na expectativa de vida entre ricos e pobres ainda seja acentuada: cerca de 15 anos. ${ }^{1}$ Uma vez que a taxa de mortalidade tenha decrescido em virtude das doenças infectocontagiosas, a maioria das pessoas, atingindo a idade adulta, morre em decorrência de doenças crônico-degenerativas, como doença cardiovascular, certos tipos de câncer e diabetes, quer vivam em países desenvolvidos ou não. ${ }^{1,2}$

Muitas das doenças crônicas associadas à idade e redução na capacidade funcional podem ser prevenidas através do aumento da capacidade cardiovascular e da força muscu- 
lar, através de programas de treinamento para a aptidão cardiorrespiratória e de treinamento resistido. ${ }^{4}$ Por conseguinte, a prescrição de exercícios resistidos em idosas possibilita benefícios por apresentarem características mais próximas das atividades do cotidiano, principalmente pelo aumento da massa, força e potência muscular, estimulando, com isso, a maior participação em atividades físicas, além daquelas desenvolvidas nos programas de treinamento institucionalizados, tendo como resultado o aumento da independência ${ }^{4}$ e, consequentemente, da habilidade de realizar as atividades da vida diária.

Assim, a prescrição e orientação do exercício físico para pessoas idosas devem estabelecer alterações positivas no círculo vicioso do envelhecimento, onde este processo leva à inatividade física e à consequente redução na aptidão física e aumento da fragilidade músculo-esquelética, resultando num estilo de vida cada vez mais dependente. Esta dependência ocasiona uma menor motivação e uma baixa autoestima, aumentando a ansiedade e depressão, ocasionando o aumento ainda maior da inatividade física. ${ }^{5}$

Este estudo teve como objetivo analisar as possíveis alterações no perfil lipídico e da glicose sanguínea e nos indicadores da aptidão física de mulheres acima de 60 anos fisicamente ativas e inativas.

\section{METODOLOGIA}

A população foi constituída de mulheres com idades variando entre 60 e 81 anos de idade residentes em Curitiba. A amostra foi constituída de 23 mulheres, sendo 12 no grupo de fisicamente ativas (GA), ou seja, que participam de um programa de exercícios físicos monitorados, há pelo menos seis meses, na Clínica de Atividade Física e Qualidade de Vida Mayoredad; e 11 no grupo inativo (GI), ou seja, aquelas que não participem regularmente em esportes e recreações programadas ou de atividades físicas moderadas e vigorosas segundo os critérios estabelecidos no IPAQ, versão curta; ${ }^{6}$ ou de exercícios físicos monitorados, há pelo menos seis meses, selecionadas aleatoriamente na comunidade. Foi aplicado o questionário da AHA/ACSM Facilitado de Pré-participação em Saúde e Aptidão, ${ }^{7}$ para a estratificação dos riscos à saúde.

Os valores de colesterol total (CT) e triglicérides sanguíneos (TG) foram obtidos mediante o monitor portátil Accutrend ${ }^{\circledR}$ GCT (Roche Diagnostics), e a glicose sanguínea foi mensurada com o monitor portátil Accu-Chek ${ }^{\circledR}$ Advantage (Roche Diagnostics), que utilizam o método de fotometria de reflexão.

Foram coletados dados da massa corporal e estatura, os perímetros da cintura e do quadril; calcularam-se o índice de massa corporal (IMC) e a relação cintura-quadril, RCQ. O valor da densidade corporal (D) foi obtido através da equação para mulheres de Tran \& Weltman. ${ }^{8}$ Para o percentual de gordura $(\% \mathrm{G})$, usou-se Brozek et al.: ${ }^{9} \% \mathrm{G}$ $=((4,57 / \mathrm{D})-4,142) \times 100$. A massa de gordura (MG, kg) foi obtida pela equação: 
$\mathrm{MG}, \mathrm{kg}=(\% \mathrm{G} / 100) \times$ Massa Corporal, $\mathrm{kg}$. A massa corporal magra (MCM, kg) foi obtida subtraindo-se a MG da MC, onde $\mathrm{MCM}, \mathrm{kg}=\mathrm{MC}, \mathrm{kg}-\mathrm{MG}$, kg.

A aptidão cardiorrespiratória foi obtida mediante o Canadian Aerobic Fitness Test, conforme descrito em Jetté et al. ${ }^{10}$ Para a aptidão muscular, utilizaram-se os testes de força de membros propostos por Rikli \& Jones ${ }^{11}$ (teste de sentar e levantar em 30s, para a força de membros inferiores; teste de flexão de cotovelo em 30s, para a força de membros superiores) e de força abdominal proposto pelo ACMS. ${ }^{12}$

Os escores do teste de agilidade/equilíbrio foram obtidos pelo teste 8-Ficar em Pé e Andar, conforme descrito previamente. ${ }^{11} \mathrm{Um}$ registro alimentar de três dias foi utilizado para descrever o perfil alimentar. ${ }^{13}$ Todos os participantes consentiram em participar do estudo, conforme Termo de Consentimento de Participação aprovado pelo Comitê Setorial de Ética em Pesquisa do Setor de Ciências Biológicas da Universidade Federal do Paraná registrado no CEP-Biológicas sob o número 008-05.

A normalidade dos dados foi constatada pelo teste de Shapiro-Wilks e, para comparar as médias entres os grupos, utilizouse o teste $t$ de Student independente, no pacote estatístico $R$ for Windows versão 1.9.1, sendo $\mathrm{p}<0,05$.

\section{RESULTADOS}

Todas as mulheres ativas faziam uso de medicamentos prescritos no momento da coleta dos dados. Somente $36,3 \%$ das mulheres inativas informaram não fazer uso de medicamento. As classes de medicamentos mais utilizados foram: os anti-hipertensivos, para o tratamento do hipotireoidismo, osteoporose e depressão/ansiedade.

As características antropométricas e da composição corporal de mulheres idosas com mais de 60 anos ativas e inativas são mostradas na tabela 1 .

Mulheres ativas apresentaram médias de idade e de estatura significativamente superiores ( $\mathrm{p}<0,05)$, em relação às mulheres inativas. Os perímetros da cintura e do quadril, a RCQ e a G\% foram similares entre os grupos ( $p>0,05)$. O grupo de mulheres ativas apresentou valores de MG e de MCM superiores, mas não significativos $(p>0,05) \mathrm{em}$ relação ao grupo de mulheres inativas.

Seis mulheresativas apresentaram valoresabaixo do limiar para diagnóstico de gordura abdominal $(0,85),{ }^{14}$ eseis mulheres apresentaram valores acima deste limiar. No grupo de mulheres inativas, seis apresentaram valores abaixo do limiar e cinco estavam acima. Mulheres idosas ativas e inativas apresentaram valores similares de $\mathrm{G} \%$. As idosas ativas possuíam valores de $\mathrm{MGe}$ MCM superiores, mas não significativos, ( $p$ > $0,05)$ do que as idosas inativas (Tabela 1). 
Tabela 1 - Características antropométricas, de composição corporal de mulheres idosas com mais de 60 anos ativas e inativas. Curitiba, 2007.

\begin{tabular}{|c|c|c|c|}
\hline & Ativas $(\mathrm{n}=12)$ & Inativas $(\mathrm{n}=11)$ & $\mathrm{p}$ \\
\hline Idade (anos) & $71,58 \pm 5,41$ & $65,90 \pm 4,01^{*}$ & 0,009 \\
\hline Massa corporal $(\mathrm{kg})$ & $70,42 \pm 8,23$ & $66,50 \pm 10,17$ & 0,319 \\
\hline Estatura $(\mathrm{cm})$ & $159,41 \pm 4.59$ & $154,09 \pm 6,22^{*}$ & 0,028 \\
\hline IMC $\left(\mathrm{kg} / \mathrm{m}^{2}\right)$ & $27,77 \pm 3,60$ & $27,97 \pm 3,72$ & 0,897 \\
\hline \multicolumn{4}{|l|}{ IMC (\%) } \\
\hline $18,5-24,9$ & 33,33 & 18,18 & - \\
\hline $25,0-29,9$ & 41,66 & 45,45 & - \\
\hline $30,0-34,9$ & 25,00 & 27,27 & - \\
\hline $35,0-39,9$ & 0 & 0 & - \\
\hline$>40$ & 0 & 0 & - \\
\hline Perímetro Cintura $(\mathrm{cm})$ & $88,55 \pm 5.01$ & $87,13 \pm 10,22$ & 0,682 \\
\hline Perímetro do Quadril (cm) & $103,75 \pm 6.13$ & $102,60 \pm 11,16$ & 0,759 \\
\hline $\mathrm{RCQ}, \mathrm{cm}$ & $0,85 \pm 0,04$ & $0,84 \pm 0,07$ & 0,856 \\
\hline$\% \mathrm{G}$ & $42,78 \pm 3,53$ & $42,11 \pm 6,38$ & 0,754 \\
\hline MG, kg & $30,33 \pm 5,69$ & $28,48 \pm 8,60$ & 0,546 \\
\hline $\mathrm{MCM}, \mathrm{kg}$ & $40,08 \pm 3,16$ & $38,01 \pm 3,47$ & 0,149 \\
\hline
\end{tabular}

$* \mathrm{p}<0,05$

Com relação ao perfil qualitativo alimen$\operatorname{tar}$ (Tabela 2), as idosas ativas e inativas não se diferenciaram significativamente $(\mathrm{p}>$ $0,05)$, no percentual de carboidratos, lipídios e proteínas consumidos.

Quanto ao perfil metabólico, mulheres ativas mostraram valores de colesterol total, triglicérides e glicose sanguínea inferiores ao das inativas (Tabela 2), embora estas di- ferenças não tenham sido significativas, $\mathrm{p}$ $>0,05$. Com respeito à glicemia, mulheres idosas ativas apresentaram valores inferiores aos das inativas, mas não significativos $(p>0,05)$.

Com respeito à glicemia, mulheres idosas ativas apresentaram valores inferiores aos das inativas, mas não significativos $(\mathrm{p}>0,05)$. 
Tabela 2 - Perfil metabólico das mulheres com mais de 60 anos ativas e inativas. Curitiba, 2007.

\begin{tabular}{lccc}
\hline & Ativas $(\mathrm{n}=12)$ & Inativas $(\mathrm{n}=11)$ & $\mathrm{p}$ \\
\hline Metabólico $(\mathrm{mg} / \mathrm{dl})$ & & & \\
Colesterol total & $179,66 \pm 22,19$ & $192,40 \pm 31,79$ & 0,282 \\
Triglicérides & $201,00 \pm 105,18$ & $208,33 \pm 109,23$ & 0,883 \\
Glicose & $104,91 \pm 19,75$ & $116,72 \pm 23,39$ & 0,120 \\
\hline
\end{tabular}

Nos testes de aptidão física, as idosas ativas apresentaram valores significativamente superiores $(\mathrm{p}<0,05)$, somente no teste de 8 -ficar em pé e andar que mulheres as inativas (Tabela 3).

As mulheres inativas apresentaram valores ligeiramente superiores na flexão de cotovelo, no abdominal parcial e no
VO2max do que os valores mostrados pelas inativas, embora estas diferenças não foram significativas, $\mathrm{p}>0,05$. As idosas ativas mostraram melhores valores de força de membros inferiores (teste de sentar e levantar), embora não significativo ( $p>0,05)$, o que pode refletir no melhor desempenho no teste de agilidade/ equilíbrio.

Tabela 3 - Testes de aptidão física das mulheres idosas com mais de 60 anos ativas e inativas. Curitiba, 2007.

\begin{tabular}{lccc}
\hline \multicolumn{1}{c}{ Teste } & Ativas $(\mathrm{n}=12)$ & Inativas $(\mathrm{n}=11)$ & $\mathrm{p}$ \\
\hline 8 Ficar em pé e andar $(\mathrm{s})$ & $5,79 \pm 1,06$ & $7,63 \pm 2,27^{*}$ & 0,028 \\
Sentar e levantar (rep) & $17,16 \pm 3,43$ & $14,45 \pm 5,50$ & 0,166 \\
Flexão de cotovelo (rep) & $21,00 \pm 4,97$ & $23,09 \pm 4,01$ & 0,282 \\
Abdominal parcial (rep) & $29,08 \pm 4,77$ & $30,63 \pm 11,49$ & 0,683 \\
VO2max ml $/ \mathrm{kg} / \mathrm{min}$ & $18,78 \pm 2,61$ & $20,15 \pm 2,31$ & 0,245 \\
\hline
\end{tabular}

$* \mathrm{p}<0,05 ; \mathrm{s}=$ tempo em segundos; rep $=$ numero de repetições

Conforme o teste de aptidão cardiorrespiratória (VO2max) proposto por Jetté et al. ${ }^{10} \mathrm{e}$ utilizando os valores de referência propostos, tanto as ativas como as inativas apresentaram um nível de aptidão abaixo da média $(20-24 \mathrm{ml} / \mathrm{kg} / \mathrm{min})$, para ambos os grupos.

\section{DISCUSSÃO}

O tema sobre a atividade física em idosos tornou-se preocupação constante tanto de órgãos internacionais, como Colégio Americano de Medicina Esportiva, ${ }^{3}$ quanto nacionais, como no caso brasileiro, $\mathrm{da}$ 
Sociedade Brasileira de Medicina do Esporte em conjunto com a Sociedade Brasileira de Geriatria e Gerontologia. ${ }^{5}$ Mostra-se, portanto, a importância dos estudos relacionados ao envelhecimento, tanto no que diz respeito aos marcadores de estratificação de risco à saúde, que podem ser diferentes do de pessoas mais jovens, assim como na utilização de terapias, particularmente a atividade física, na prevenção e tratamento de doenças relacionadas ao processo etário.

Inicialmente, devemos citar algumas limitações do estudo. O número da amostra foi pequeno, o que inviabiliza extrapolar os resultados, sendo necessário realizar maior pesquisa com respeito às condições de saúde e os níveis de atividade física da população idosa brasileira. Além disso, o colesterol total, os triglicérides e a glicemia foram obtidos mediante monitores portáteis, sem respeitar um mínimo de 12 horas de jejum, o que pode dificultar a comparação com os valores de corte para a estratificação de risco coronariano propostos.

O IMC, como indicador de sobrepeso e obesidade, e o perímetro da cintura, como indicador de distribuição de gordura corporal, vêm sendo correlacionados à causa principal de óbitos em idosos, particularmente com relação ao risco cardiovascular. ${ }^{15-20}$ A nossa amostra apresentou um alto risco para a doença coronariana quando se leva em consideração tanto o ponto de corte para o IMC (>25 kg/m2) quanto o posto de corte para o perímetro da cintura $(>88 \mathrm{~cm})$ e o consumo de medicamentos; $66.6 \%$ das idosas ativas e $72.7 \%$ das idosas inativas apre- sentam sobrepeso (IMC > $>25 \mathrm{~kg} / \mathrm{m} 2)$; e $50 \%$ das idosas ativas e $45.4 \%$ das idosas inativas apresentaram perímetros de cintura $>88$ $\mathrm{cm}$. Além disso, nos dois grupos houve uma alta incidência no consumo de medicamentos, principalmente os anti-hipertensivos. As idosas ativas não diferiram das idosas inativas em relação ao IMC, ao contrário de Brach et al..$^{21}$ mas similarmente aos de Kanaya et al. ${ }^{22}$

Apesar do risco aumentado à saúde quando se considera o ponto de corte para o IMC $\left(>25 \mathrm{~kg} / \mathrm{m}^{2}\right)$ proposto pela Organização Mundial da Saúde,,${ }^{14}$ há a possibilidade de valores de estratificação de risco diferenciados quando se consideram aquele indicador e o perímetro da cintura em conjunto. Ardern et al. ${ }^{15}$ procuraram estabelecer valores indicativos para o perímetro da cintura em função das categorias de IMC e sua associação com os indicativos limiares de $88 \mathrm{~cm}$ para mulheres e $102 \mathrm{~cm}$ para os homens, para predizer os riscos de eventos coronarianos. Esses autore ${ }^{15}$ concluíram que os valores indicativos estáticos para o perímetro da cintura de homens e mulheres são insuficientes para identificar o aumento no risco à saúde. Assim, eles propõem limiares específicos do perímetro da cintura em função das categorias do IMC. Estes pontos de corte se situam em 90, 100, 110 e $125 \mathrm{~cm}$ para os homens; e 80, 90, 105, $115 \mathrm{~cm}$ para as mulheres, em função das categorias propostas pelo IMC: peso normal, sobrepeso, obesidades I, II e III, respectivamente.

Quando aplicamos na amostra deste estudo os pontos de corte propostos por Ar- 
dern et al. ${ }^{15}$ para o perímetro da cintura em função da categoria do IMC descrito acima, 50\% das idosas ativas e nenhuma das idosas inativas apresentariam risco à saúde Da mesma forma, alguns dados sugerem que os pontos corte utilizados para pessoas mais jovens não seriam adequados para pessoas idosas com respeito ao risco de infarto do miocárdio. Dey et al..$^{23}$ propuseram pontos de corte para o aumento do risco de infarto de $>99 \mathrm{~cm}$ para homens e $>94 \mathrm{~cm}$ para mulheres idosas. Quanto ao IMC, $28 \mathrm{~kg} / \mathrm{m}^{2}$ foi o ponto de corte proposto para o aumento do risco de infarto, com associação significativa somente para os homens. Assim, quando consideramos o ponto de corte $>94$ $\mathrm{cm}$, apenas $8,33 \%$ das idosas ativas e 18,18\% das inativas teriam risco aumentado de infarto contra $50,0 \%$ e $45,45 \%$ das ativas e inativas, respectivamente, quando se considera o ponto de corte de $88 \mathrm{~cm} .{ }^{14}$

Apesar de não haver diferença significativa no \%G, na MG e na MCM, pode-se sugerir que a maior MCM mostrada pelo grupo de ativas tenha um potencial de proteção contra as causas de óbitos em idosos. Bigaard et al. ${ }^{17}$ mostraram uma associação oposta entre o IMC e a massa de gordura corporal e a massa livre de gordura em homens e mulheres de 50 a 64 anos, e que as duas últimas são preditores independentes para as todas as causas de mortalidade. Kyle et al. ${ }^{24}$ mostraram que a atividade física teve efeito positivo na prevenção do aumento na MG, e no IMC com o avanço da idade, num grupo com idades entre 15 e 64 anos. Esses autores concluíram que a atividade física tem efeito positivo na prevenção do aumento da massa de gordura e no IMC com a idade. Toth et al..$^{25}$ publicaram um artigo de revisão sobre os efeitos do exercício aeróbio ou do exercício resistido na composição corporal em idosos ( $>55$ anos) e concluíram que o exercício aeróbio é efetivo para a redução na massa de gordura, mas não aumenta a massa livre de gordura e está relacionado com o número de sessões realizadas. Já o exercício resistido é efetivo para a redução da massa de gordura e para o aumento na massa livre de gordura.

Não foram encontradas diferenças estatisticamente significativa no perfil alimentar e no perfil metabólico entre mulheres ativas e inativas. Os valores aqui demonstrados são similares aos de outro estudo com a população brasileira. ${ }^{26}$ Vale ressaltar que o consumo de gorduras foi menor que $30 \%$ nos dois grupos (ativas e inativas), confirmando as recomendações da Associação Americana de Cardiologia ${ }^{27}$ no tratamento e prevenção do ganho de peso em adultos. Isto sugere que o menor consumo médio de gorduras pode ter influenciado os valores de colesterol total, já que o hábito dietético tem importante influência na variação das concentrações de LDL-colesterol e no HDL-colesterol..$^{26} \mathrm{Da}$ mesma forma, o exercício físico tem importantes implicações quando associado ao consumo dietético nos perfis antropométricos. Ross et al., ${ }^{28}$ ao realizaram estudo com o objetivo de determinar os efeitos independentes da dieta ou do exercício na redução do peso e da resistência à insulina mulheres (3952 anos) moderadamente obesas (IMC = 
33,2 ), mostraram que em quatro grupos (dieta, dieta combinada com exercício aeróbio, exercício aeróbio e controle), o exercício combinado à restrição dietética tem maiores efeitos sobre a gordura total $(-6.7$ versus $-4.1 \mathrm{~kg}$, $\mathrm{p}<0.001)$, na gordura abdominal (-1.7 versus $-0.9 \mathrm{~kg}, \mathrm{p}<0.001)$ e na melhoria da aptidão cardiorrespiratória $(-0.5$ versus $+0.5 \mathrm{~L} /$ $\min , \mathrm{p}<0.008)$ que o grupo de dieta somente. Esses autores também demonstraram que o grupo de dieta combinada com o exercício aeróbio reduziu a resistência insulina em $32 \%$, quando comparado ao grupo controle $(p<0.008)$, e que esta melhora na resistência à insulina pode ser explicada pela maior redução na gordura total e abdominal apresentada por este grupo.

Apesar de os valores médios de colesterol total e triglicérides apresentarem valores próximos aos considerados "normais", ${ }^{29}$ Chyou et al..$^{30}$ argumentam que os valores de estratificação de risco cardíaco podem ser mais elevados do que o preconizado em pessoas mais jovens; além disso, há uma clara tendência da associação apenas entre o HDL-colesterol ou da razão HDL/colesterol total e o risco de mortalidade.

No presente estudo, não se encontraram diferenças significativas no perfil lipídico e glicêmico entre as mulheres ativas e inativas. Contudo, Pescatello \& Murphy ${ }^{10,31,32}$ mostraram que há uma associação negativa entre os lipídios e glicose plasmática e os níveis de atividade física.

Somente no teste de agilidade/equilíbrio (8-Ficar em pé andar) houve diferença signifi- cativa entre o grupo de idosas ativas e o grupo de idosas inativas. Vale salientar que, apesar de não se encontrarem diferenças entre as demais variáveis da aptidão física, houve diferença significativa na faixa etária entre os grupos (grupo ativo $>$ grupo inativo). Isto pode sugerir que, com o aumento da atividade física, é possível minimizar os efeitos do envelhecimento mesmo após os 60 anos. Apovian et al. ${ }^{33}$ ao analisarem o efeito da massa corporal na função física de mulheres idosas, mostraram que o excesso de massa corporal, predito pelo IMC, pode prejudicar certos aspectos $\mathrm{da}$ função física, particularmente na parte superior do corpo, onde a força e a coordenação não são influenciadas.

Outros autores têm mostrado que o nível de aptidão pode ser influenciado pelas atividades diárias e pelo treinamento, tanto o aeróbio quanto o resistido, utilizando diferentes metodologias e intensidades e tendo importantes implicações no que diz respeito ao risco à saúde, perfil lipídico e à independência em idosos. ${ }^{21,34}$ Stathokostas et al. ${ }^{35}$ verificaram que o VO2max diminui com a idade, embora as mulheres apresentem razão de declínio menor. Nós demonstramos que, apesar da diferença na média etária, as mulheres ativas eram cinco anos mais velhas na média, não havendo diferenças significativas na força muscular e no VO2max.

\section{CONCLUSÃO}

Considerando as limitações deste estudo, conclui-se que a performance física pode ser mantida durante o processo de envelhecimen- 
to, através de um programa de exercícios orientados. Participar de um programa de exercícios pode tanto prevenir quanto reduzir os efeitos deletérios relacionados ao envelhecimento, dentre as quais as doenças crônico-degenerativas, como hipertensão, diabetes e as doenças coronarianas e seus fatores de risco, como os

\section{REFERÊNCIAS}

1. Kalache A, Veras RP, Ramos LR. O envelhecimento da população mundial: um desafio novo. Rev Saúde Pública 1987; 21:200-10.

2. Veras RP, Ramos LR, Kalache A. Crescimento da população idosa no Brasil: transformações e consequências na sociedade. Rev Saúde Pública 1987; 21: 225-33.

3. ACSM-American College of Sports Medicine. Position stand - exercise and physical activity for older adults. Med Sci Sports Exerc 1998; 30: 992-1008.

4. Hunter GH, McCarthy JP, Bamman MM. Effects of Resistance Training on Older Adults. Sports Med 2004; 34: 329-48.

5. Nóbrega ACL, Freitas EV, Oliveira MAB, Leitão M B, Lazzoli JK, Nahas RM, et al. Posicionamento Oficial da Sociedade Brasileira de Medicina do Esporte e da Sociedade Brasileira de Geriatria e Gerontologia: Atividade Física e Saúde no Idoso. Revista brasileira de medicina do esporte 1999; 5: 207-11.

6. CELAFISCS. Questionário Internacional de Atividade Física (IPAQ).[ acesso 2006 fev] http://www.celafiscs.com.br

7. ACSM/AHA - American College of Sports Medicine - American Heart Association. Joint position statement: recommendations for cardiovascular perfis lipídicos e glicêmicos; a redução da massa muscular; o aumento da massa de gordura; a redução da capacidade funcional, demonstrada pela redução da independência e autonomia; tornando-se um dos importantes fatores para a melhora da saúde e da qualidade de vida desta população.

screening, staffing, and emergency policies at health / fitness facilities. Med Sci Sports Exerc 1998; 30: 1009-18.

8. Tran ZV, Weltman A. Generalized equation for predicting body density of women from girth measurements. Med Sci Sports Exerc 1989; 21: 101-4.

9. Brozek J, Grande F, Anderson JT, Keys A. Densidometric analysis of body composition: revision of some quantitative assumptions. Ann N Y Acad Sci 1963; 110: 113-40.

10. Jetté M, Campbell J, Mongeon J, Routhier $\mathrm{R}$. The Canadian Home Fitness Test as a predictor of aerobic capacity. Can Med Assoc J 1976; 114: 680-2.

11. Rikli RE, Jones CJ. Development and validation of a functional test for community-residing older adults. J Aging Phys Act 1999; 7: 129-61.

12. ACSM - American College of Sports Medicine. Diretrizes do ACSM para os testes de esforço e sua prescrição. Rio de Janeiro: Guanabara-Koogan; 2003.

13. Dunn C. Avaliação nutricional. In: Tritschler $\mathrm{KA}$, organizador. Medida e avaliação em educação física e esportes. São Paulo: Manole; 2003. p. 489-526.

14. WHO Consulation on Obesity. Obesity: Prevention and Managing: The Global 
Epidemic. Report of a WHO Consultation on Obesity; 1997.

15. Ardern CI, Janssen I, Ross R, Katzmarzyk PT. Development of health-related waist circunference thresholds within bmi categories. Obes Res 2004; 12: 1094-103.

16. Bigaard J, Tjonneland A, Thomsen BL, Overvad K, Heitmann BL, Sorensen TIA. Waist circunference, BMI, smoking, and mortality in middle-aged men and women. Obes Res 2004; 11: 895-903.

17. Bigaard J, Frederikesn K, Tjonneland A, Thomsen BL, Overvad K, Heitmann BL, et al. Body fat and fat-free mass and all-cause mortality. Obes Res 2004; 12: 1042-9.

18. Dey DK, Lissner L. Obesity in 70-year-old subjects as a risk factor for 15-year coronary heart disease incidence. Obes Res 2003; 11: 817-27.

19. Iwao S, Iwao N, Muller DC, Elahi D, Shimokata H, Andres R. Effect of aging on the relationship between multiple risk factors and waist circunference. J Am Geriatr Soc 2000; 48: 788-94.

20. Yan LL, et al. BMI and Health-Related Quality of Life in Adults 65 Years and Older. Obes Res 2004; 12: 69-76.

21. Brach, JS, Simonsick EM, Kritchevsky S, Yaffe K, Newman AB. The association between physical function and lifestyle activity and exercise in health, aging and body composition study. J Am Geriatr Soc 2004; 52: 502-9.

22. Kanaya AM, Vittinghoff E, Shlipak MG, Resnick HE, Visser M, Grady D, et al. Association of total and central obesity with mortality in postmenopausal women with coronary heart disease. Am J Epidemiol 2003; 158: 1161-70.

23. Dey DK, Rothenberg E, Sundlh V, Bosaeus I, Steen B. Waist circumference, body mass index, and risk for stroke in older people: a 15-year longitudinal population study of 70-year-olds. J Am Geriatr Soc 2002; 50: 1510-8.

24. Kyle UG, Gremion G, Genton L, Slosman DO, Golay A, Pichard C. Physical activity and fat-free and fat mass by bioelectrical impedance in 3853 adults. Med Sci Sports Exerc 2001; 33: 576-84.

25. Toth MJ, Beckett T, Pohelman ET. Physical activity and the progressive change in body composition with aging: current evidence and research issues. Med Sci Sports Exerc 1999; 31: S590-6.

26. Fornés NS, Martins IS, VelásquesMeléndez G, Latorre MRDO. Escores de consumo alimentar e níveis lipêmicos em população de São Paulo, Brasil. Rev Saúde Pública 2002; 36: 12-8.

27. Kraus RM, Eckel RH, Howard B, Appel LJ, Daniels SR, Deckelbaum RJ, et al. AHA Dietary Guidelines - Revision 2000: A statement for healthcare professionals from the Nutrition Committee of the American Heart Association. Circulation 2000; 102: 2284-99.

28. Ross R, Janssen I, Dawson J, Kungl AM, Kuk JL, Wong L, et al. Exercise-Induced Reduction in Obesity and Insulin Resistance in Women: a randomized controlled trial. Obes Res 2004; 12: 789-98.

29. Santos RD. III Diretrizes brasileiras sobre dislipidemias e diretriz de prevenção da aterosclerose do Departamento de Aterosclerose da Sociedade Brasileira de Cardiologia. Arq Bras Cardiol 2001; 77: 1-48.

30. Chyou P, Eaker ED. Serum cholesterol concentrations and all-cause mortality in older people. Age Ageing 2000; 29: 69-74.

31. Pescatello LS, Murphy D. Lower intensity physical activity is advantageous for fat distribution and blood glucose among 
viscerally obese older adults. Med Sci Sports Exerc 1998; 30: 1408-13.

32. Pescatello LS, Murphy D, Costanzo D. Low-intensity physical activity benefits blood lipids and lipoproteins in older adults living at home. Age Ageing 2000; 29: 433-9.

33. Apovian CM, Frey CM, Wood GG, Rogers JZ, Still CD, Jensen GL. Body Mass Index and Physical Function in Older Women. Obes Res 2002; 10: 740-7.

34. Adams KJ, et al. Progressive strength training in sedentary, older African American women. Med SciSports Exerc 2001; 33: 1567-76.

35. Stathokostas L, Jacob-Jonhson S, Petrella RJ, Paterson DH. Longitudinal changes in aerobic power in older men and women. J Appl Physiol 2004; 97: 784-9.

Recebido: 4/8/2008

Revisado: 15/5/2009

Aprovado: 19/5/2009 